\title{
The Cause-and-Effect Link Between Obesity and Insulin Resistance
}

\author{
Hamid Alizadeh ${ }^{1, *}$ \\ ${ }^{1}$ School of Physical Education, Mazandaran University, Babolsar, Mazandaran, Iran \\ "Corresponding author: School of Physical Education, Mazandaran University, Babolsar, Mazandaran, Iran. Email: h.alizadeh.aw@gmail.com \\ Received 2021 June 14; Accepted 2021 June 21.
}

Keywords: Insulin Resistance, Obesity, Cause-and-Effect Link

\section{Dear Editor,}

Insulin resistance (IR) is characterized as higher circulating insulin levels which make target cells like skeletal muscle, fat, and liver cells take glucose out of the blood and enter it into cells (1). Although factors such as genetics, ageing, and ethnicity are considered for developing IR, there are other IR-inducing agents like excessive body fat, too much belly fat, lack of sleep and exercise, and smoking (2).

There has been much interest in the relationship between obesity and IR. In this letter, I intend to discuss the cause-and-effect relationship between obesity and IR. There is an ongoing and intense discussion on which one comes first, obesity or IR.

Insulin hormone is in charge of reducing blood glucose (3). When it is said that someone is insulin resistant, it means that their main metabolic cells like skeletal muscle, fat, and liver are not able to grab glucose out of the bloodstream and use it as their fuels, subsequently increasing blood glucose levels (4). Increased blood glucose level is known as non-diabetic hyperglycemia or prediabetes (4). If this condition persists, it can lead to diabetes which brings about serious health problems (5). Therefore, clarifying the mentioned question seems to be of great importance to help us understand the way type 2 diabetes develops. Additionally, by knowing how something occurs, we can be informed of its treatment and even prevention alternatives. In this letter, I will discuss four perspectives on the field of cause-and-effect relationship between obesity and IR.

The commonly accepted perspective on the underlying mechanisms of type 2 diabetes states that IR occurs as the result of obesity. In other words, this viewpoint considers weight gain as the cause of IR. Insulin action (insulin sensitivity) and insulin secretion (beat cell-secreting function) are subject to impairment, which is induced by overeating
(6). The insulin-secreting function of the pancreas is negatively associated with a fat threshold (6). Once one's fat levels go beyond their fat threshold, diabetes type 2 develops. The twin cycle hypothesis states the etiology of type 2 diabetes. Based on this hypothesis, chronic consumption of high-calorie foods leads to liver fat accumulation, which in turn spills over into the pancreas. Both liver and pancreas cycles reinforce each other's effects, which ultimately result in hyperglycemia, hyperinsulinemia, and type 2 diabetes (6). Altogether, this point of view on the cause-andeffect relationship between obesity and IR considers obesity as the cause of IR.

However, Dr. Peter Attia puts forward the idea that it is IR that drives obesity and weight gain. He believes that the cause-and-effect relationship between IR and obesity has been considered the wrong way round. Dr. Peter Attia's hypothesis suggests that chronically consuming highcarbohydrate foods over time slowly cause the IR's underlying mechanisms to trigger. Based on this hypothesis, when IR reaches a given threshold, weight gain and obesity can easily happen. Therefore, Peter Attia strongly considers IR as the cause of obesity (7).

Another viewpoint is proposed by Professor Roger Unger. Professor Unger explains that fat accumulation in the pancreas kills beta cells and makes alpha cells insulin resistant that suppresses insulin spike, and results in glucagon increase. This perspective considers overeating (weight gain) as the cause of IR and type 2 diabetes, except that the whole process is a result of glucagon hormone. Studies show that when the glucagon receptor is removed from mice, type 2 diabetes or IR does not develop in mice (7).

The last interesting perspective was proposed by Dr. Malcom Kendrick. He believes that there will always be exceptions to any hypothesis, and he takes the examples of Sumo wrestlers and Berardinelli-Seip lipodystrophy (people with almost no fat cells) as the exceptions to the general 
agreement that higher fat is equal to a higher risk of diabetes. Sumo wrestlers are highly unlikely to develop type 2 diabetes, and $100 \%$ of Berardinelli-Seip lipodystrophy people have type 2 diabetes. Dr. Kendrick believes that diabetes (IR) is mainly a problem with energy storage. If energy excess has a place to be stored, neither insulin nor blood glucose levels will increase. Accordingly, Dr. Kendrick believes that it is IR that causes weight gain (7).

Conclusions: Based on these four different viewpoints, diabetes seems to be a much more complicated condition than the commonly held view of gaining weight causes IR to happen. Given the proposed hypotheses, it seems that IR coupled with glucagon action can cause weight gain and obesity.

\section{Footnotes}

Authors' Contribution: The whole work belongs to the corresponding author.

Conflict of Interests: The author declared no conflict of interests.
Funding/Support: No funding was received.

\section{References}

1. Yaribeygi H, Farrokhi FR, Butler AE, Sahebkar A. Insulin resistance: Review of the underlying molecular mechanisms. J Cell Physiol. 2019;234(6):8152-61. doi: 10.1002/jcp.27603. [PubMed: 30317615].

2. American Diabetes Association. Standards of medical care in diabetes-2017 abridged for primary care providers. Clin Diabetes. 2017;40(Suppl 1):5.

3. van Niekerk G, Christowitz C, Conradie D, Engelbrecht AM. Insulin as an immunomodulatory hormone. Cytokine Growth Factor Rev. 2020;52:34-44. doi:10.1016/j.cytogfr.2019.11.006. [PubMed: 31831339].

4. Wallace AS, Wang D, Shin JI, Selvin E. Screening and Diagnosis of Prediabetes and Diabetes in US Children and Adolescents. Pediatrics. 2020;146(3). doi: 10.1542/peds.2020-0265. [PubMed: 32778539]. [PubMed Central: PMC7461138].

5. Beverly JK, Budoff MJ. Atherosclerosis: Pathophysiology of insulin resistance, hyperglycemia, hyperlipidemia, and inflammation. J Diabetes. 2020;12(2):102-4. doi: 10.1111/1753-0407.12970. [PubMed: 31411812].

6. Taylor R. Type 2 diabetes and remission: practical management guided by pathophysiology. J Int Med. 2021;289(6):754-70.

7. Willner T. Does insulin resistance cause weight gain? 2021. Available from: https://www.secondnature.io/. 